\title{
CHARACTERISTICS OF ELECTRON-COOLED BUNCHED BEAMS PREDICTED BY A SIMULATION
}

\author{
M. Takanaka, RIKEN, Japan
}

\section{Abstract}

Characteristics of electron-cooled bunched ion beams have been studied using the particle-tracking simulation in which the space-charge force and the force induced through the transverse broadband impedance are taken into account. The beams become space-charge dominated during the bunching of coasting beams. The simulation results show that the beams meet the transverse instability which can be cured by a transverse feedback, and the seed of the instability is the toroid field of the electron-cooling section. The following RF-wave-form distortion phenomena can be seen at stable or stabilized bunched beams. As well as the longitudinal line density becomes parabolic-liked, the nearer to the bunch center, the larger the beam size is. The bunch motion is a quasi-elastic motion instead of the synchrotron oscillation in the longitudinal direction, and is a dipole-mode coherent oscillation with the head-tail-mode number 1 in the horizontal direction.

\section{INTRODUCTION}

The Radioisotope Beam Factory (RIBF) [1] will have an electron-RI beam collider (e-RI Collider). We have studied ion-beam bunching at low energies for collision experiments. After the previous simulation [2], we found that on the way of the bunching the transverse beam density is extremely high on the very small central area, and that the mesh structure with $101 \times 101$ cells over $80 \times 80 \mathrm{~mm}^{2}$ for calculation of the transverse space-charge field is not sufficient for exact description of the field. The previous simulation results are ones misguided by using the mesh structure. We have modified the mesh structure to have the first $101 \times 101$ cells over $60 \sim 80 \times 60 \sim 80 \mathrm{~mm}^{2}$ and the second $13 \times 13$ cells at the first center cell.

In the particle-tracking simulation, we have taken the following sources of force acting on particles into account:

- Longitudinal monopole space-charge impedance, when the transverse charge distribution is round and Gaussian with one-dimensional standard deviation $\sigma$ which is much smaller than the inner radius of the vacuum chamber wall $b$ :

$$
\begin{gathered}
\left(\frac{Z_{\|}}{p}\right)_{s p}=i \frac{g(r)}{2 \beta \gamma^{2}} Z_{0}, \\
g(r) \approx-\int_{x=\frac{r^{2}}{2 \sigma^{2}}}^{\infty} \frac{e^{-x}}{x} d x+2 \log \left(\frac{b}{r}\right),
\end{gathered}
$$

where $Z_{0}$ is impedance of the free space.

- Transverse space-charge field including the influence of the chamber wall.

- Transverse dipole broad-band impedance based on the broad-band impedance model with the shunt impedance $R_{s}=\frac{2}{b^{2}} Q\left|\left(\frac{Z_{\|}}{p}\right)_{b b}\right| \frac{\omega_{r}}{\omega_{0}}$, where the effective transverse broad-band impedance $\left|\left(\frac{Z_{\|}}{p}\right)_{b b}\right|$ is assumed to be $50 \Omega, \mathrm{Q}=1, \omega_{r}=c / b$, and $\omega_{0}$ is the revolution angular frequency.

- Electron-cooling force [3].

- Solenoid field $B_{0}$ of the electron-cooling section.
- Beam twist due to the toroid field at both ends of the electron-cooling section. Ions are let pass in parallel with the $s$ axis through the toroid of which the vertical cross-section is equal to that of the solenoid. The integration of the $x$-axis component of the field along the path is approximately

$$
B_{0} x_{t}\left(\log r_{t}-\log x_{t}\right)+B_{0} x,
$$

where the toroid radial center is at $(x, s)=\left(x_{t}, 0\right)$, and the radius of the curvature of the outside inner wall is $r_{t}$. The first term expresses the effect to bend beams vertically which is compensated by using steering magnets, and the second term expresses an effect to twist the beams.

- A constantly focusing section and the drift electroncooling section of which we assume the ring lattice to consist.

- RF field for beam bunching.

Using 40,000 macro-particles, we simulated $\mathrm{U}_{238}^{92+}$ ion beams of $4 \mathrm{~mA}$, or $5.4 \times 10^{6}$ ions per bunch at $150 \mathrm{MeV} / \mathrm{u}$. Since our interest was a single bunch phenomenon, we assumed that every bunch behaves the same along the azimuthal direction. We used parameters listed in Table 1 as input data to the simulation. The simulation showed that the longitudinal and transverse electron-cooling times are $6 \mathrm{~ms}$ and $18 \mathrm{~ms}$ for a faint-current beam, respectively.

We show the simulation results from the next section.

Table 1: Parameters of the ring and the electron cooling. Ring

\begin{tabular}{lc} 
Circumference $2 \pi r$ & $180 \mathrm{~m}$ \\
Operation betatron tunes $\nu_{h} / \nu_{v}$ & $4.4 / 3.8$ \\
Momentum compaction factor & $\nu_{h}^{-2}$ \\
Chromaticities $\xi_{h} / \xi_{v}$ & $-25 /-30$ \\
Beta functions $\beta_{h} / \beta_{v}$ & $r / \nu_{h} / r / \nu_{v}$ \\
Dispersion $\eta_{h}$ & $r / \nu_{h}^{2}$ \\
Twiss parameters at the cooling section & 0 \\
$\quad \alpha_{h}^{e c}=\alpha_{v}^{e c}$ & $7 \mathrm{~m}$ \\
$\quad \beta_{h}^{e c}=\beta_{v}^{e c}$ & 60 \\
RF harmonics, or number of bunches $h$ & $4 \mathrm{~cm}$ \\
Inner radius of the vacuum chamber $b$ & $10^{-3}$ \\
\multicolumn{2}{c}{ Coasting beam } \\
Momentum spread (sixfold rms) & $10^{-6} \pi \mathrm{m} \cdot \mathrm{rad}$ \\
Rms transverse emittances $\epsilon_{h}=\epsilon_{v}$ & $4 \mathrm{~A}$ \\
\multicolumn{2}{c}{ Electron cooling } \\
Cathode temperature $k T_{c}$ & $0.1 \mathrm{eV}$ \\
Length of the cooling section & $3 \mathrm{~m}$ \\
Electron-beam radius at the section & $25 \mathrm{~mm}$ \\
Solenoid field $B_{0}$ & $0.1 \mathrm{~T}$
\end{tabular}

\section{BEAM BUNCHING}

We have the RF system which supplies a fundamental RF voltage and a third harmonic RF voltage. The system is located at the azimuth $\pi$ from the cooling section since the momentum spread can be the smallest at the section in 


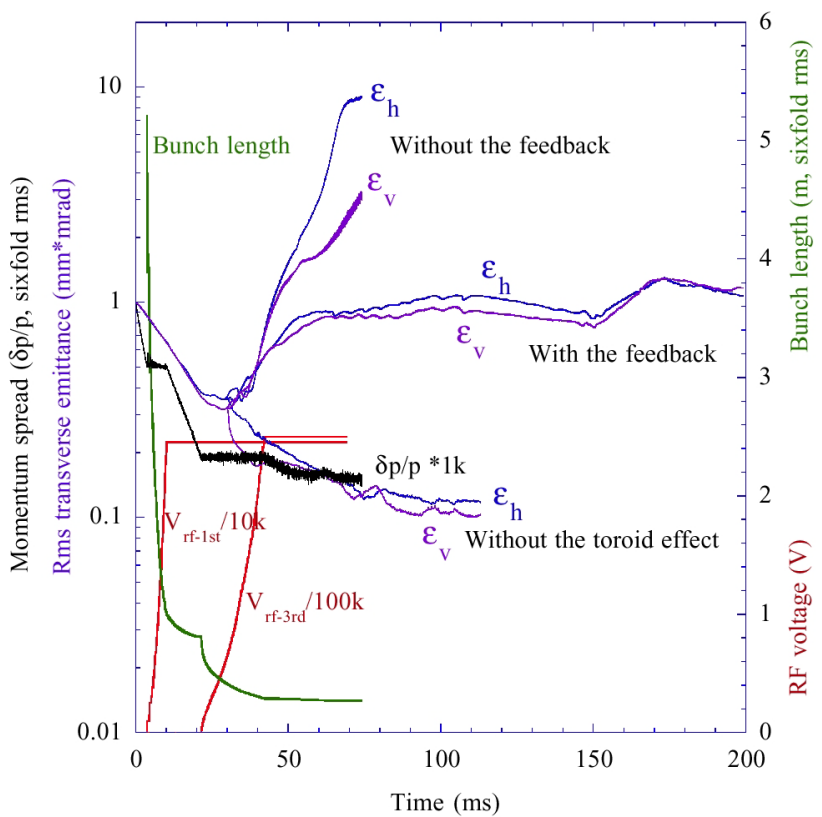

Figure 1: Evolution of the bunching of a $\mathrm{U}_{238}^{92+}$ ion beam of $4 \mathrm{~mA}$. The curves of the momentum spread and the RF voltages are for the case without the transverse feedback.

the location case. The evolution of the beam bunching is shown in Fig. 1. The coasting beam is cooled to the momentum spread $4.5 \times 10^{-4}$ at first. Then, the fundamental $\mathrm{RF}$ voltage is increased in such a way that the momentum spread is maintained at $4.5 \times 10^{-4}$. When the sixfold-rms bunch length reaches $1 \mathrm{~m}$, the increase stops. When the length reaches $0.7 \mathrm{~m}$ or most of the beam stays within onethird bunch spacing, the third harmonic RF voltage is increased up to $250 \mathrm{kV}$ holding the momentum spread. After the RF voltage $250 \mathrm{kV}$, the instantaneous beam current at the bunch center is $105 \sim 110 \mathrm{~mA}$. In the case where the transverse feedback is not used, the beam meets a transverse instability, and the beam loss starts at $60 \mathrm{~ms}$ since part of the beam hits the inner chamber wall.

In order to cure the transverse instability, we use a following transverse feedback. Picked up at the end of the cooling section where the dispersion is zero, the horizontal and vertical beam-position signals along a bunch are fed to the bunch downstream the phase advance $\pi / 2$ with the feedback gains $0.8 / \beta_{h}$ and $0.8 / \beta_{v}$, respectively. The Figure 1 shows that the feedback starts at $30 \mathrm{~ms}$ and is effective for the cure. The equilibrium momentum spread is $5 \times 10^{-5}$.

We neglect only the toroid effect in the simulation in order to check the instability source, although the neglect breaks continuity of the magnetic field from the solenoid. Figure 1 shows that the transverse emittances are damped after the neglect starts at $30 \mathrm{~ms}$.

We can describe the thin-lens approximation of a toroid twist force as a delta-function-liked kicker in terms of a Fourier series of azimuth functions. Then, the ion's motion equation due to the twist force along the ring is

$\frac{d^{2} y}{d \theta^{2}}+\nu_{v}^{2} y=\sum_{i} \epsilon_{i} x_{0} \cos \nu_{h} \theta \cos i \theta, \quad 2 \epsilon_{0}=\epsilon_{i}(i \neq 0)$,

where $i$ is an integer. The equation means that there is an emittance-increasing resonance if $\nu_{h}+\nu_{v}=i$. This holds good too, even when we consider both twist forces at the ends. We are led to a conclusion that the toroid effect is the seed of the transverse instability.

\section{TRANSVERSE INSTABILITY}

The transverse instability begins to grow up around 30 $\mathrm{ms}$ in the case without the feedback. Figure 2 shows the ion distribution at $44 \mathrm{~ms}$ at the end of the cooling section. The transverse coherent oscillation occurs around the bunch center.

Figure 3 shows the spectrum of the horizontal dipole moment at $44 \mathrm{~ms}$. The main coherent oscillation has the frequency tune 4.4 equal to the operation tune and has the head-tail mode of the so-called rigid bunch. But, the coherent oscillation has a small component with the head-tail mode number 1 of which the frequency tune is 1 . We refer to the component later.

In general it is effective for curing a transverse instability to make the chromaticity negative and smaller below transition energy. But, the chromaticity control is not effective for curing the above instability, because it makes tune spread and helps beams be trapped into the resonances.

\section{BEAMS UNDER THE TRANSVERSE FEEDBACK}

Figure 4 shows the ion distribution at $200 \mathrm{~ms}$ at the end of the cooling section. The nearer to the bunch center, the larger the betatron amplitudes are on the whole. This can be explained as follows. The longitudinal monopole space-charge impedance is the largest at the radial position 0 , and become smaller as the radial position increases. For ions with the smaller amplitudes, the stronger the RFwave-form distortion is. The ions can be farther from the bunch center than those with large amplitudes at a given small momentum spread.

Figure 5 shows the longitudinal space-space distributions at the end of the cooling section and just after the $\mathrm{RF}$ section at $200 \mathrm{~ms}$. Just before and after the RF section, the distribution is one with the opposite slop in the longitudinal phase space. The projected momentum spread just after the RF section becomes five times larger than at the cooling section. The bunch length becomes the longest at the RF section and the shortest at the cooling section. The longitudinal space-charge effect makes such a quasi-elastic motion of the bunch at the frequency tune 1 in longitudinal direction. At the same time, as we assume the same dispersion along the ring except for the cooling section, in the horizontal direction the bunch has oscillation with the head-tail mode number 1 and the frequency tune 1 due to
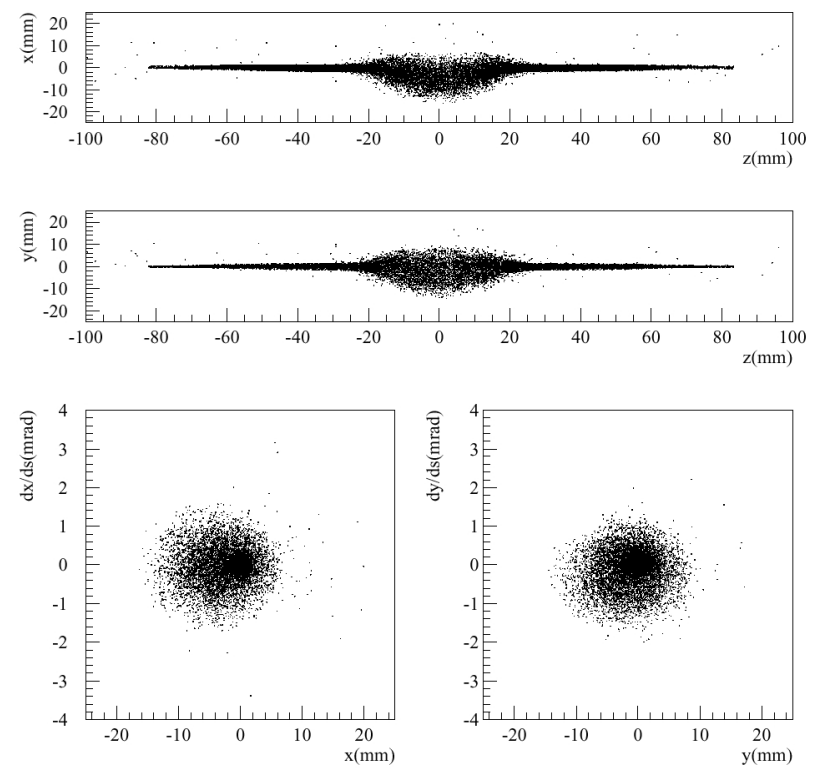

Figure 2: Ion distribution at $44 \mathrm{~ms}$ in the case without the transverse feedback. 

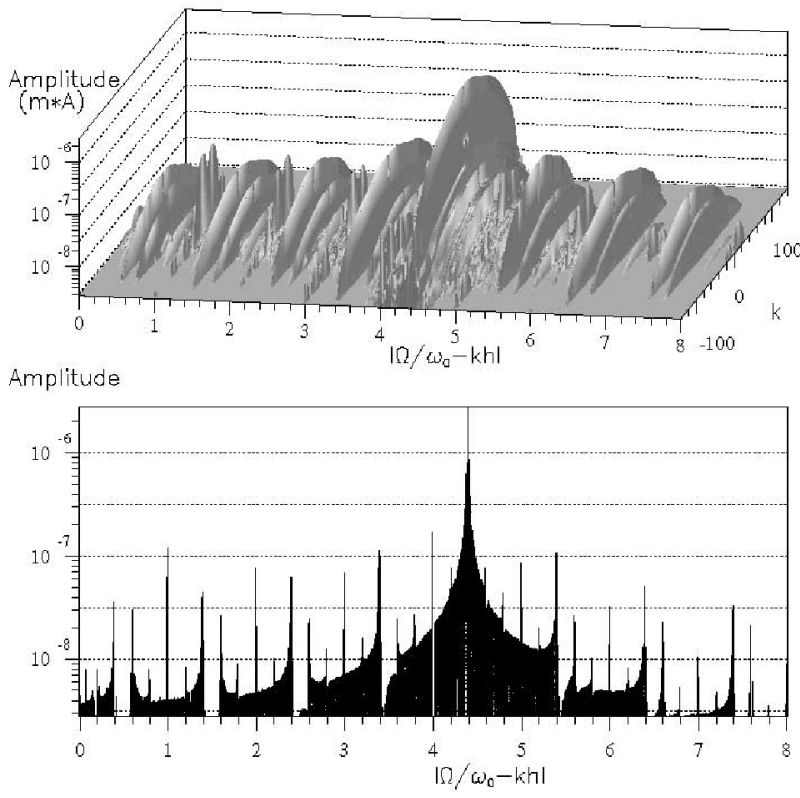

Figure 3: Spectrum of the horizontal dipole moment at 44 $\mathrm{ms}$ in the case without the transverse feedback. The spectrum below $3 \times 10^{-9} \mathrm{~m} \cdot \mathrm{A}$ is cut away.
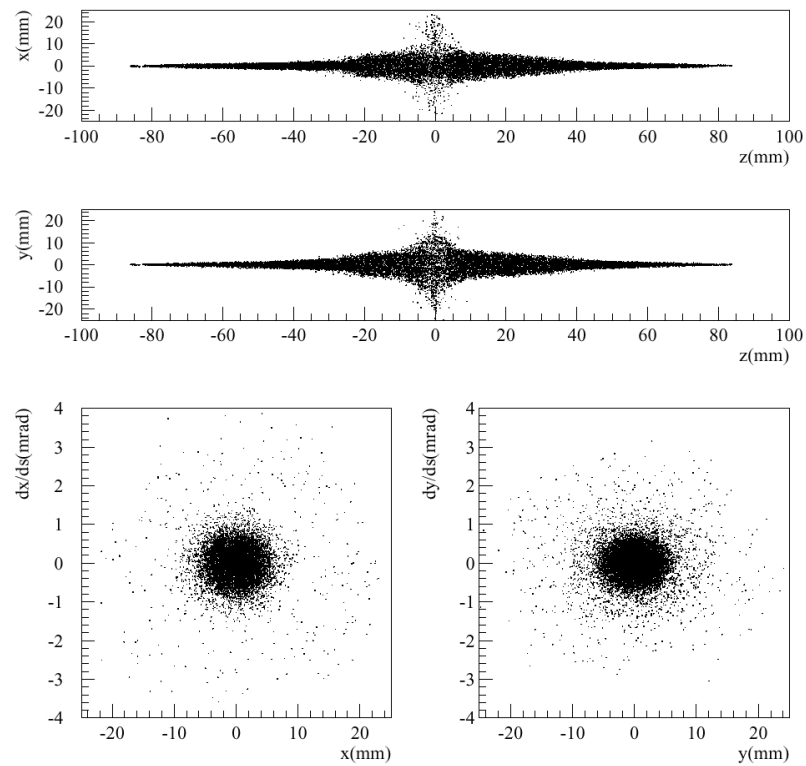

Figure 4: Ion distribution at $200 \mathrm{~ms}$ in the case with the transverse feedback.

the momentum oscillation. There is still a microwave structure on the parabolic-liked profile.

Figure 6 shows the betatron and synchrotron-tune distributions at $200 \mathrm{~ms}$. The betatron-tune shift is small for the central part of the bunch, but is large for the front and tail parts of the bunch. This means that the charge density is smaller on the central part than on the front and tail parts. The resonance line $\nu_{h}+\nu_{v}=8$ crosses the tune-distribution area at the side near to the operation tune point. When the operation tune point was changed to $(4.45,3.90)$ farther from the resonance line, the simulation showed that the transverse emittances become less than $7 \times 10^{-7} \pi \mathrm{m} \cdot \mathrm{rad}$.

Under the third harmonic RF voltage $250 \mathrm{kV}$ the bare synchrotron tune is 0.083 . Figure 6 shows that most of ions
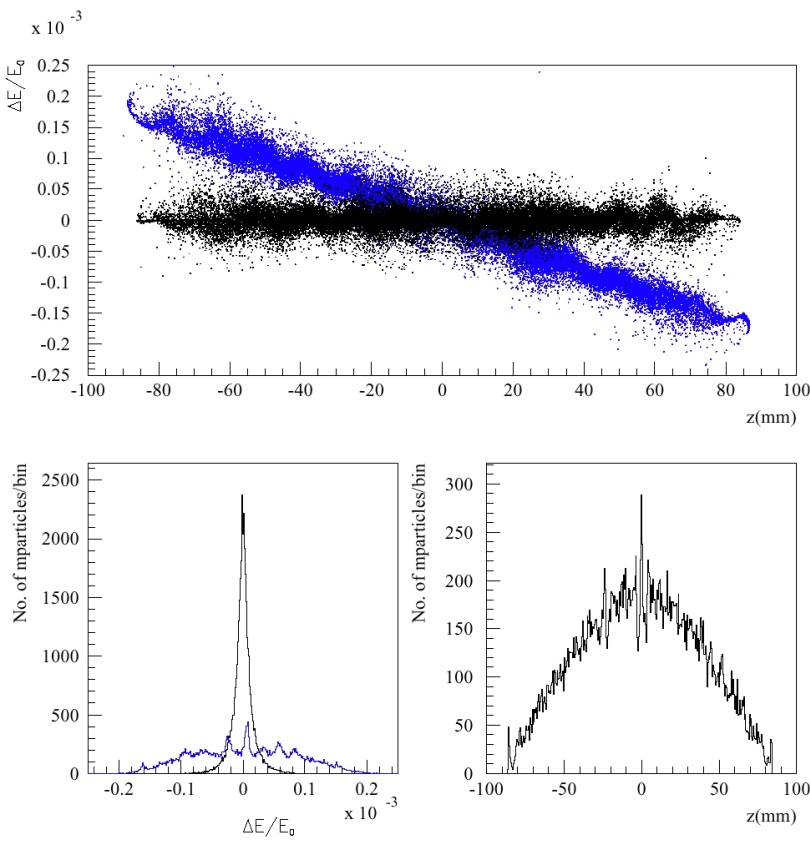

Figure 5: Longitudinal phase-space distribution at $200 \mathrm{~ms}$ in the case with the transverse feedback. In the upper, the slanted distribution is one just after the RF system, and the non-slanted distribution is one at the end of the cooling section. In the lower right, the projected distribution is one at the end of cooling section.

have nearly the tune 0 and part of ions in the central region have large tunes which are less than the bare tune.
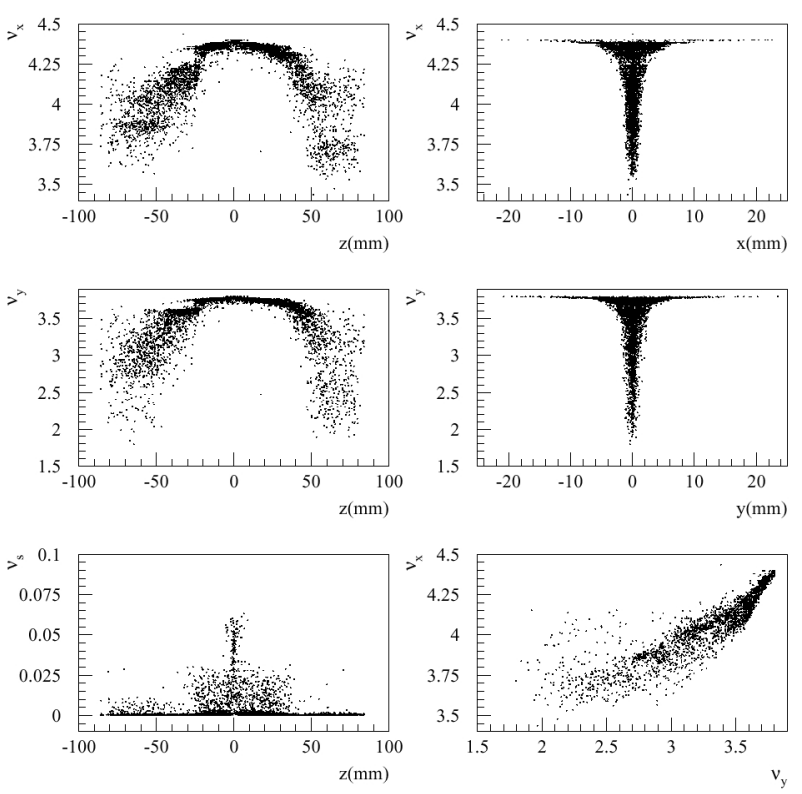

Figure 6: Tune distributions at $200 \mathrm{~ms}$ in the case with the transverse feedback.

\section{REFERENCES}

[1] Y.Yano et al, RIKEN Acc. Progress Report (2000) 301.

[2] M.Takanaka, T.Katayama, PAC'99, 1719.

[3] I.N.Meshkov, Phys. Part. Nucl. 25 (6), (1994) 631. 